\title{
Comparison of Phenotypic and Genotypic Patterns of Antimicrobial-Resistant Bacteroides fragilis Group Isolated from Healthy Individuals in Vietnam and
} Japan

\author{
Hanh Vu (iD) \\ Masahiro Hayashi ${ }^{2,3}$ \\ Thang Nam Nguyen ${ }^{4}$ \\ Diep Thi Khong ${ }^{4}$ \\ Hoa Thi Tran ${ }^{4}$ \\ Yoshimasa Yamamoto (iD) \\ Kaori Tanaka ${ }^{\text {I-3 }}$ \\ 'United Graduate School of Drug \\ Discovery and Medical Information \\ Sciences, Gifu University, Gifu City, Gifu, \\ Japan; ${ }^{2}$ Life Science Research Center, Gifu \\ University, Gifu City, Gifu, Japan; \\ ${ }^{3}$ Institute for Glyco-core Research \\ (iGCORE), Gifu University, Gifu City, \\ Gifu, Japan; ${ }^{4}$ Center for Medical and \\ Pharmaceutical Research and Service, \\ Thai Binh University of Medicine and \\ Pharmacy, Thai Binh City, Thai Binh, \\ Vietnam
}

Purpose: Normal non-pathogenic flora can harm the host by acting as a reservoir of resistance determinants that are potentially transferable to human pathogens. This study aimed to assess the phenotypic and genotypic antimicrobial susceptibility patterns of the Bacteroides fragilis group (BFG) isolated from healthy individuals in Vietnam and Japan in order to elucidate the prevalence of antimicrobial resistance in human flora in the two economically and geographically different countries.

Materials and Methods: BFG was isolated from fecal samples of 80 healthy individuals in Vietnam $(n=51)$ and Japan $(n=29)$. Isolated strains were identified using MALDI-TOF MS, and the minimum inhibitory concentration (MIC) of 18 antibiotics was determined using the agar dilution method. Additionally, 20 antimicrobial resistance genes were detected using standard PCR.

Results: A total of 139 BFG strains belonging to 11 BFG species were isolated from the two countries, with diversity in the prevalence of each species. B. fragilis was not the predominant species. Isolations from Vietnam and Japan showed some similarities in terms of $\mathrm{MIC}_{50}$ values, $\mathrm{MIC}_{90}$ values, and the percentage of resistant strains. However, isolations from Vietnam showed significantly higher resistance to piperacillin, cefmetazole, clindamycin, tetracycline, and minocycline. ErmB, tet36, tetM, nim, catA, and $q n r \mathrm{~A}$ were not found in either country. CepA was more common in B. fragilis than in non-fragilis Bacteroides. In contrast, $c f \mathrm{~A}, \operatorname{erm} \mathrm{G}, m e f \mathrm{~A}, m s r \mathrm{SA}, \operatorname{tet} \mathrm{X}, \operatorname{tet} \mathrm{X} 1, \operatorname{bex} \mathrm{A}, q n r \mathrm{~B}$, and $q n r \mathrm{~S}$ were found only in non-fragilis Bacteroides. There were differences in the prevalence of erm $\mathrm{G}$, $\operatorname{lin} \mathrm{A}, m e f \mathrm{~A}$, $m s r \mathrm{SA}$, and qnrS between isolates from Vietnam and Japan.

Conclusion: This study is the first report on the antimicrobial susceptibility patterns in the BFG isolated from healthy individuals in Vietnam and Japan. Compared to isolations from Japan, isolations from Vietnam showed significantly higher resistance to antimicrobial agents. The distribution of various antibiotic resistance genes also differed between the two countries.

Keywords: antimicrobial resistance patterns, resistance genes, human flora

\section{Introduction}

Anaerobic bacteria, including the most frequently isolated Bacteroides fragilis group (BFG) strains, are normal resident members of the gastrointestinal microbiota. BFG members are human opportunistic pathogens that cause severe intra-abdominal, postoperative wound, skin, and soft tissue infections along with aerobic bacteria. The
United Graduate School of Drug

Discovery and Medical Information

Sciences, Gifu University, I-I Yanagido,

Gifu City, Gifu, 50I-I 194, Japan

Tel $+8 \mathrm{I}-58-230-6554$

Fax +8I-58-230-655I

Email kktb@gifu-u.ac.jp 
number of antimicrobials that are effective against BFG bacteria is relatively limited. Increasing antibiotic resistance in BFG strains has been reported worldwide in recent years, including resistance to $\beta$-lactams, tetracyclines, macrolides, clindamycin and fluoroquinolones. ${ }^{1-6}$

The wide distribution of antibiotic resistance genes in BFG harbored by the human gastrointestinal tract could contribute to the maintenance of a balanced microbiota that protects against adverse effects of antibiotic treatments. On the other hand, BFG can potentially harm the host by acting as a reservoir of resistance determinants that may be transferable to human pathogens. ${ }^{7-9}$ Moreover, their products may protect commensal bacteria and enteric pathogens against antibiotics, such as membrane vesicles carrying surface-associated $\beta$-lactamases that protect Salmonella typhimurium against $\beta$-lactam antibiotics. ${ }^{10,11}$

Recent studies in Vietnam and Japan showed that various antibiotic resistance genes, including $\mathrm{mcr}$ and

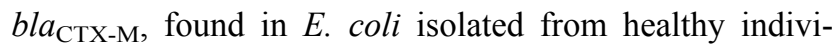
duals could be horizontally transferred among bacterial cells. ${ }^{12-14}$ These findings raised questions about the antibiotic susceptibility status and resistance genes carried by other human fecal microbiota species, especially BFG. Unfortunately, little information is available on the antibiotic resistance status and antibiotic resistance genes in BFG in healthy individuals, not only in Vietnam but also in Japan.

This study aimed to investigate the phenotypic and genotypic patterns of antimicrobial-resistant BFG isolated from healthy people in Vietnam and Japan. A wide range of antibiotics (18 antibiotics) and resistance genes (20 genes) were assessed. This research is the first of its kind for these countries and presents a model for similar research to be undertaken elsewhere.

\section{Materials and Methods}

\section{Sample Collection, Isolation, and}

\section{Identification}

Fecal samples were collected from 51 and 29 healthy individuals from Thai Binh City, Vietnam and Gifu City, Japan, respectively. All volunteers had no signs of colon or bowel inflammation or disease, and those with a history of antibiotic use during the last three months prior to the study were excluded.

Fecal samples from each person were collected in Puritan ${ }^{\circledR}$ Fecal Opti-Swab CB-206 (Puritan, USA), transported to the laboratory, and inoculated on anaerobic agar plates [Bacteroides Bile Esculin (BBE) agar; Kyokuto Pharmaceutical Industrial, Tokyo, Japan] and BBE-CAZ (BBE with $30 \mathrm{mg} / \mathrm{L}$ ceftazidime - CAZ). The plates were then incubated in an anaerobic atmosphere for $48 \mathrm{~h}$ at 37 ${ }^{\circ} \mathrm{C}$. Six well-grown colonies were selected from each plate. Each colony was cultured on modified Gifu Anaerobic Medium (GAM) agar (Nissui Pharmaceutical Co. Ltd, Tokyo, Japan) and incubated in an anaerobic atmosphere (AnaeroPack - Anaero, Mitsubishi Gas Chemical Co. Inc., Tokyo, Japan) for $48 \mathrm{~h}$ at $37{ }^{\circ} \mathrm{C}$. Isolates that grew under aerobic conditions were excluded from the study. Isolated strains were identified by matrixassisted laser desorption ionization - time of flight mass spectrometry (MALDI-TOF) (MALDI Biotyper ${ }^{\circledR}$ MBT, Bruker Japan, Yokohama, Japan). The isolates were stored in $15 \%$ skim milk at $-80^{\circ} \mathrm{C}$.

\section{Antibiotic Susceptibility Test}

Minimum inhibitory concentration (MIC) values were determined using an agar dilution method based primarily on the recommendations of the Clinical and Laboratory Standards Institute (CLSI). ${ }^{15}$ The isolates were evaluated for susceptibility to 18 antibiotics: ampicillin, piperacillin, ampicillin/sulbactam, piperacillin/tazobactam, cefmetazole, ceftriaxone, ceftazidime, cefazolin, meropenem, tetracycline, minocycline, erythromycin, clarithromycin, clindamycin, levofloxacin, ciprofloxacin, sulfamethoxazole, and metronidazole. B. fragilis ATCC 25285 and B. thetaiotaomicron ATCC 29741 were used as quality control strains. Brucella HK agar medium (Kyokuto Pharmaceutical Industrial Co., Ltd., Tokyo, Japan) supplemented with 5\% laked sheep blood was used as the test medium. The test strains $\left(10^{5} \mathrm{CFU} / \mathrm{spot}\right)$ were inoculated and incubated in an anaerobic chamber $\left(82 \% \mathrm{~N}_{2}, 10 \%\right.$ $\mathrm{CO}_{2}$, and $8 \% \mathrm{H}_{2}$ ) (Anaerobox, Hirasawa Ltd, Tokyo, Japan) at $35{ }^{\circ} \mathrm{C}$ for $48 \mathrm{~h}$.

\section{Detection of $\beta$-Lactamase}

Nitrocefin discs (BBL Cefinase; Becton, Dickinson, Baltimore, MD, USA) were used to detect $\beta$-lactamase production according to the manufacturer's instructions. Isolates with $\beta$-lactamase activity will show induce a yellow to red color change on the area where the isolate was smeared. Isolates without $\beta$-lactamase activity were scored as negative if there was no change in the color of the nitrocefin disc after 30 minutes. 


\section{PCR-Based Detection of Resistance Genes}

The following 20 antimicrobial genes were investigated: серA, cfxA, cfiA ( $\beta$-lactamase), ermB, ermF, ermG, linA, mefA, $\quad m s r S A$ [macrolide-lincomycin-streptogramin (MLS) resistant], tet $M$, tet $Q$, tet $X$, tet $X 1$, tet36 (tetracycline resistant), bexA, qnrA, qnrB, qnrS (quinolone resistant), nim (metronidazole resistant), and catA (chloramphenicol resistant). For those strains found to harbor $c f \mathrm{~A}$, further investigation of three insertion sequences (IS1186, IS1187, and IS942) was carried out.

Bacterial cells from the colonies on the surface of anaerobic agar plates were suspended in $0.5 \mathrm{~mL}$ water in $1.5 \mathrm{~mL}$ Eppendorf tubes and incubated at $95^{\circ} \mathrm{C}$ for $8 \mathrm{~min}$. The supernatants of the centrifuged suspensions (5 min, $16,000 \times \mathrm{g})$ were used as template DNA and stored at $-20{ }^{\circ} \mathrm{C}$. The template DNA $(5 \mu \mathrm{L})$ was amplified in a 45 $\mu \mathrm{L}$-reaction mixture consisting of $25 \mu \mathrm{L}$ of $2 \mathrm{x}$ Quick Taq ${ }^{\circledR}$ HS DyeMix (Toyobo Co. Ltd, Osaka, Japan), $2 \mu \mathrm{L}$ of each primer, and $16 \mu \mathrm{L}$ of distilled water. Amplification was performed using a Takara PCR Thermal Cycler Dice TP 600 (Takara Bio Inc., Shiga, Japan). The PCR conditions used to detect all 20 genes and 3 insert sequences, primer sequences, and PCR parameters are listed in Table S1. The PCR products were examined by electrophoresis on a $2 \%$ agarose gel.

\section{Statistical Evaluation}

Comparison of the antimicrobial-resistant percentage and prevalence of different genes between $B$. fragilis and nonfragilis Bacteroides, and between the two countries were evaluated using a chi-squared test. The statistical significance was set at $p<0.05$.

\section{Results}

\section{Overview of the Isolates}

All the isolates (6 isolates per sample per individual, except isolates that grew under aerobic conditions) were tested for susceptibility to 18 antimicrobial agents and for the presence of 20 antibiotic-resistance genes to build their antimicrobial-resistant profiles. If many isolates in the same person belonged to the same species, only isolates with different profiles were chosen for analysis. Finally, 76 BFG strains from Vietnam and 63 strains from Japan were selected. The identified isolates are listed in Table 1. Eleven BFG species were identified in this study. The first, second, and third most common species were
Table I Identification of Isolations from Form Healthy Individuals in Vietnam and Japan

\begin{tabular}{|l|c|c|}
\hline \multirow{2}{*}{ Species } & \multicolumn{2}{|c|}{ Number of Isolations } \\
\cline { 2 - 3 } & Vietnam & Japan \\
\hline Bacteroides caccae & 1 & 0 \\
Bacteroides cellulosilyticus & 0 & 1 \\
Bacteroides fragilis & 3 & 9 \\
Bacteroides ovatus & 5 & 15 \\
Bacteroides stercoris & 9 & 5 \\
Bacteroides thetaiotaomicron & 15 & 6 \\
Bacteroides uniformis & 1 & 1 \\
Bacteroides massiliensis & 2 & 0 \\
Parabacteroides distasonis & 27 & 10 \\
Parabacteroides merdae & 0 & 2 \\
Phocaeicola vulgatus & 13 & 14 \\
\hline Total & $\mathbf{7 6}$ & 63 \\
\hline
\end{tabular}

Parabacteroides distasonis (35.5\%), B. thetaiotaomicron (19.7\%), and Phocaeicola (formerly Bacteroides) vulgatus (17.1\%) in Vietnam, and B. ovatus $(23.8 \%)$, P. vulgatus (22.2\%), and P. distasonis (15.9\%) in Japan. The difference in the prevalence of $B$. fragilis between Vietnam and Japan was significant ( $p<0.05$, Table S2). All the isolated strains (139) tested positive for $\beta$-lactamase production.

\section{Antimicrobial Susceptibility Profiles}

The susceptibility test results are presented in Table 2. Isolations from Vietnam and Japan showed some similarities in $\mathrm{MIC}_{50}$ values, $\mathrm{MIC}_{90}$ values, and percentages of resistant strains. All isolates were resistant to ampicillin, and none of the isolates were resistant to metronidazole. The rates of resistance to piperacillin, cefmetazole, clindamycin, and tetracycline were high in both countries. However, the isolates from Vietnam showed a significantly higher rate of resistance to these four agents $(p<0.05)$. The $\mathrm{MIC}_{50}$ values of three cephalosporins (cefazolin, ceftriaxone, and ceftazidime) and two macrolides (clarithromycin and erythromycin), which have no breakpoint settings, were higher than $128 \mu \mathrm{g} / \mathrm{mL}$ in both countries. The distribution pattern of minocycline was almost the same as that of tetracycline and tended towards lower MIC values. The $\mathrm{MIC}_{50}$ and $\mathrm{MIC}_{90}$ values of minocycline were $4 \mu \mathrm{g} / \mathrm{mL}$ and $16 \mu \mathrm{g} / \mathrm{mL}$ in Vietnam and 4 $\mu \mathrm{g} / \mathrm{mL}$ and $8 \mu \mathrm{g} / \mathrm{mL}$ in Japan. If the breakpoint of tetracycline was applied to minocycline, the resistance rate to minocycline was still much lower than that of tetracycline. The levofloxacin susceptibility status of the two countries 


\begin{tabular}{|c|c|c|}
\hline & 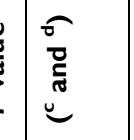 & 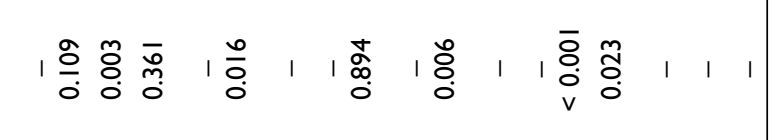 \\
\hline & 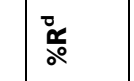 & 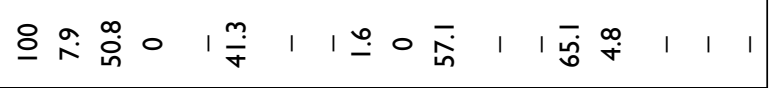 \\
\hline & $\begin{array}{l}\frac{\mathcal{J}}{\xi} \\
\frac{y_{0}}{200} \\
\frac{0}{2} \\
\frac{0}{\Sigma}\end{array}$ & 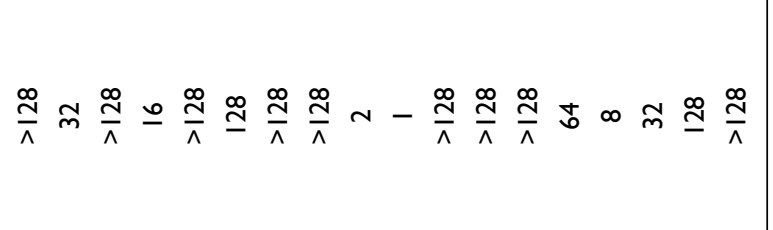 \\
\hline & 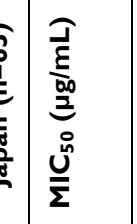 & 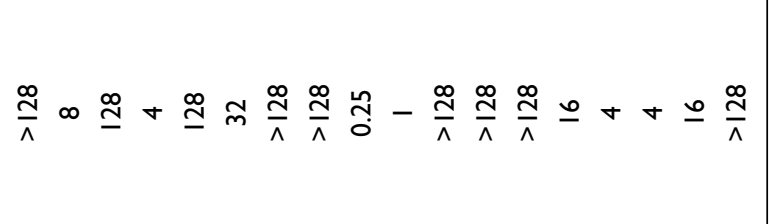 \\
\hline & 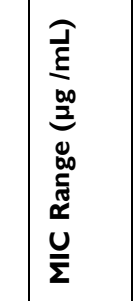 & 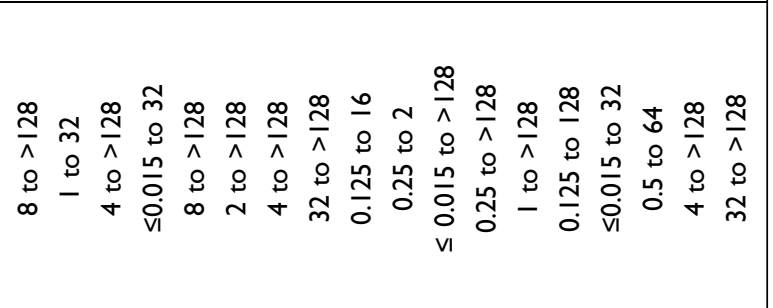 \\
\hline & 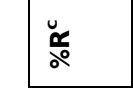 & 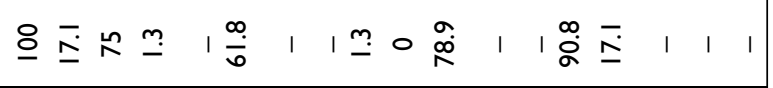 \\
\hline & 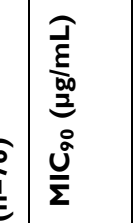 & 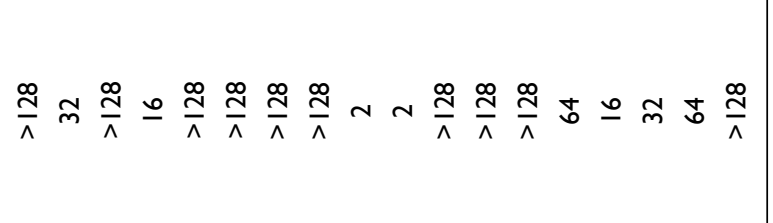 \\
\hline & 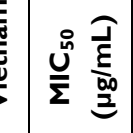 & 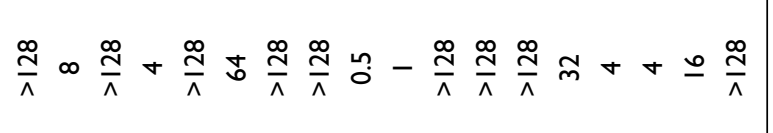 \\
\hline & 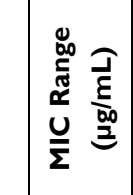 & 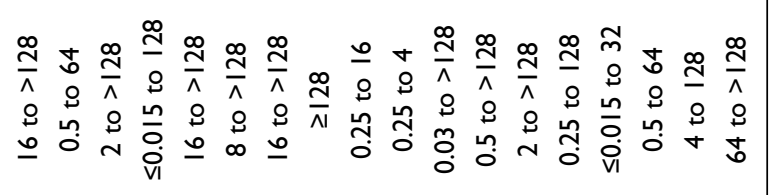 \\
\hline & 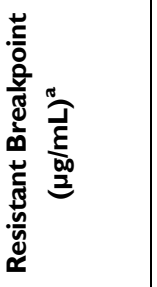 & 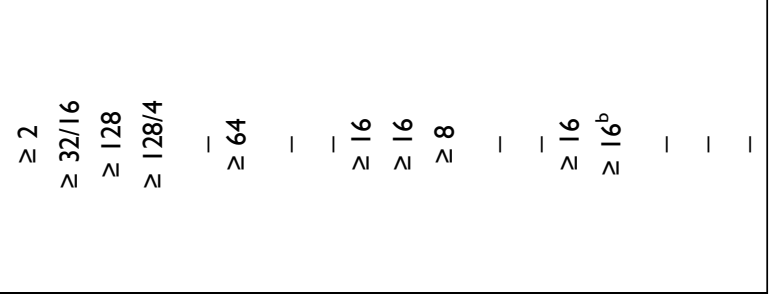 \\
\hline & 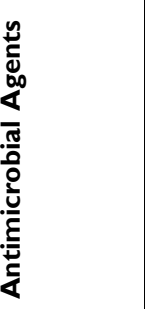 & 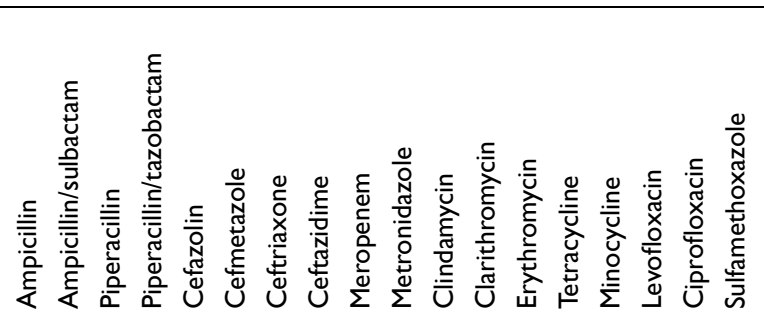 \\
\hline
\end{tabular}


was similar. However, Japan showed a higher number of strains with high MIC values of ciprofloxacin; only one strain from Vietnam had an MIC value of $128 \mu \mathrm{g} / \mathrm{mL}$, but $10(13.9 \%)$ strains from Japan had MIC values $\geq 128 \mu \mathrm{g} /$ $\mathrm{mL}$ (Table 2, Figure S1). Thus, the ciprofloxacin $\mathrm{MIC}_{90}$ of isolations from Japan was $128 \mu \mathrm{g} / \mathrm{mL}$, which was markedly higher than that of isolations from Vietnam $(64 \mu \mathrm{g} /$ $\mathrm{mL}$ ) (Table 2). All isolates were highly resistant to sulfamethoxazole.

Notably, two meropenem-resistant isolates were found in this study (one from Vietnam and one from Japan). Both isolates were non-fragilis Bacteroides (P. distasonis from Vietnam and B. ovatus from Japan) and had MIC values of $16 \mu \mathrm{g} / \mathrm{mL}$.

\section{Distribution of Resistance Genes}

The distribution of antibiotic resistance genes is presented in Table 3. All the resistance genes present in B. fragilis were also found in non-fragilis Bacteroides. In contrast, $c f \mathrm{~A}, \operatorname{erm} \mathrm{G}, m e f \mathrm{~A}, m s r \mathrm{SA}, t e t \mathrm{X}, t e t \mathrm{X} 1, \operatorname{bex} \mathrm{A}, q n r \mathrm{~B}$, and $q n r \mathrm{~S}$ were found only in non-fragilis Bacteroides. There was a significant difference in the distribution of erm $G$, lin $A$, mefA, $m s r S A$, and $q n r S$ between the two countries. The prevalence of erm $\mathrm{G}$ and $\operatorname{lin} A$ was significantly higher in Japan $(\mathrm{p}<0.001)$ and in Vietnam $(\mathrm{p}=0.003)$, respectively, than in the other country. Tet $Q$ was the most prevalent antibiotic resistance gene in both countries. All bexA genes were found in B. thetaiotaomicron.

\section{$\beta$-Lactam Resistance Genes}

Of the $12 \mathrm{~B}$. fragilis strains isolated from both countries, $11(91.7 \%)$ harbored cepA (Table 3). Only one B. fragilis strain from Japan was found to be negative for cepA, and its MIC value for ampicillin was the lowest $(16 \mu \mathrm{g} / \mathrm{mL})$. The difference in the distribution of cepA in B. fragilis and non-fragilis Bacteroides was significant $(p<0.001$, Table 3 ) in both countries. As shown in Figure 1, cepA, $c f x \mathrm{~A}$, and $c f \mathrm{~A}$ were only found in strains with ampicillin MIC values $\geq 64 \mu \mathrm{g} / \mathrm{mL}$. Nevertheless, $41 \%$ and $44 \%$ of isolates from Vietnam and Japan, respectively, were resistant to ampicillin with high MIC values and did not carry any of these genes (Figure 1). All cepA-positive isolates (15 strains, Table 3 ) were susceptible to $\beta$-lactamase inhibitor combination agents. Four strains were resistant to cefmetazole with MIC $>128 \mu \mathrm{g} / \mathrm{mL}$ (two B. thetaiotaomicron and one B. ovatus from Vietnam, and one $B$. fragilis from Japan). Among these four strains,

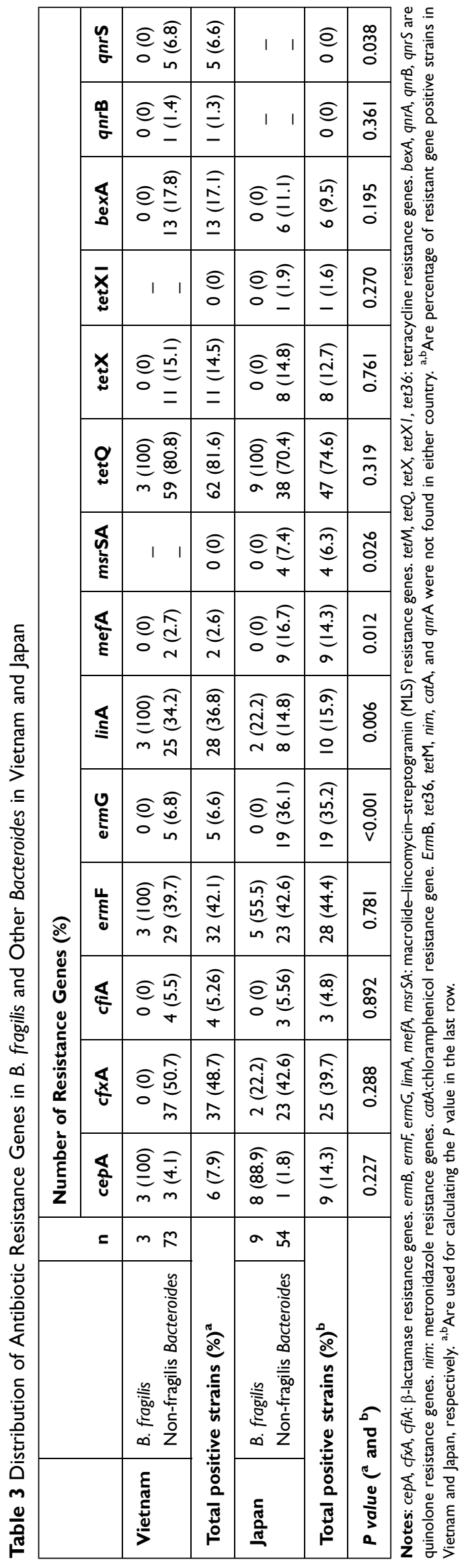




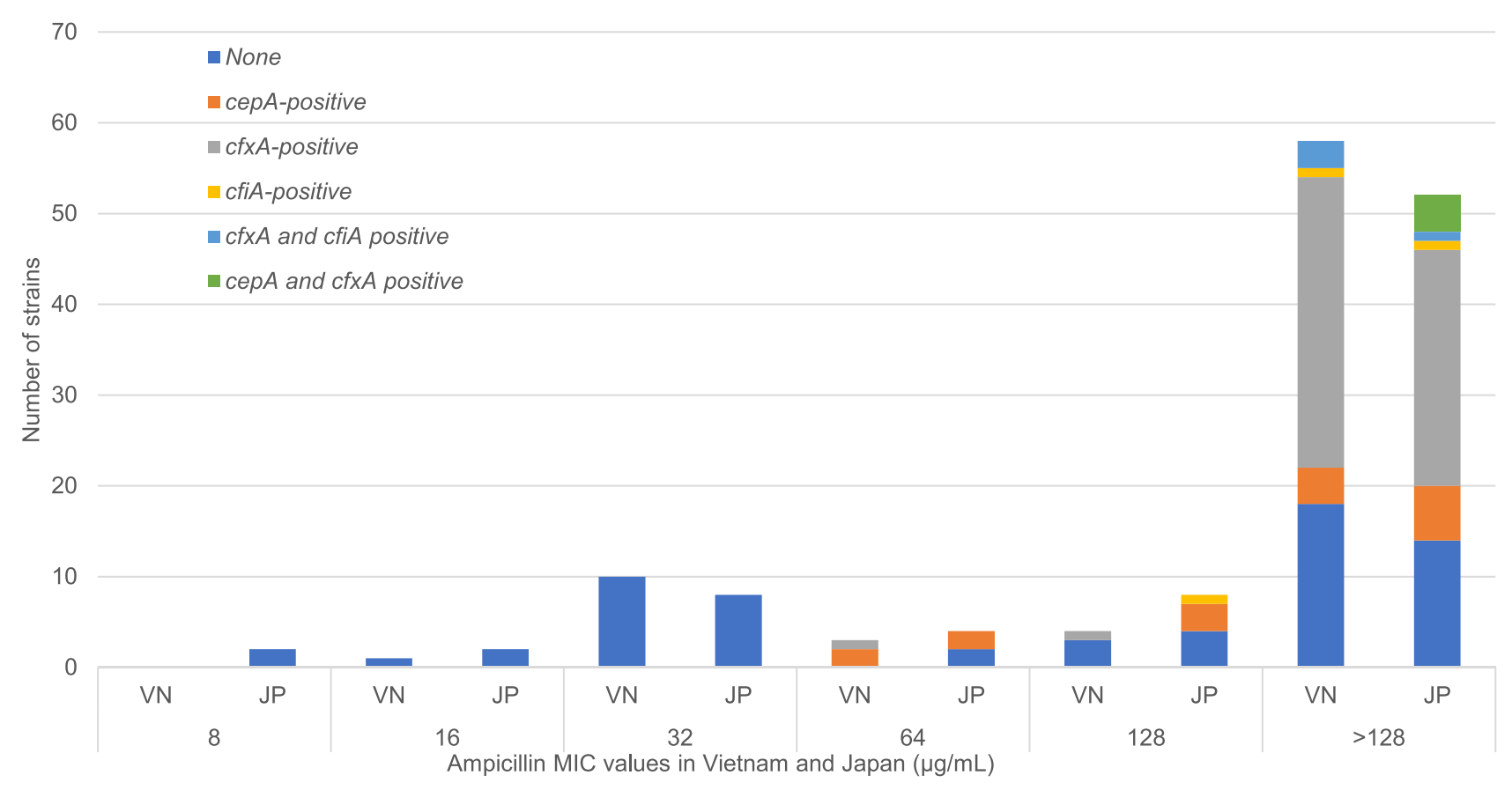

Figure I The distribution of $\operatorname{cepA}, c f x A$, and $c f \mathrm{~A}$ in BFG strains in Vietnam and Japan based on MIC values for ampicillin. Abbreviations: BFG, Bacteroides fragilis group; MIC, minimum inhibitory concentration; VN, Vietnam; JP, Japan.

B. thetaiotaomicron from Vietnam and B. fragilis from Japan did not harbor $c f x$ A or $c f i$.

Notably, two meropenem-resistant isolates were found in this study (one from Vietnam and one from Japan). Both isolates were non-fragilis Bacteroides (P. distasonis from Vietnam and $B$. ovatus from Japan) and had MIC values of $16 \mu \mathrm{g} / \mathrm{mL}$. P. distasonis did not carry cfiA, but B. ovatus did. In contrast, four strains from Vietnam and two from Japan harbored $c f i$ A but had MIC values $\leq 1 \mu \mathrm{g} / \mathrm{mL}$. These six strains were also susceptible to cephalosporins and $\beta$ lactamase inhibitor combination agents. Since the insertion of IS elements can increase the expression of the $c f i$ A genes, we checked three insertion sequences (IS1186, IS1187, and IS942), but all were negative (data not shown).

\section{Macrolide-Lincomycin-Streptogramin Resistance Genes}

In total, clindamycin-resistant strains comprised $83.3 \%$ (50/60), 70.8\% (17/24), 68.4\% (26/38), 81.8\% (9/11), and $75.0 \%(3 / 4)$ of the total erm F-, erm $\mathrm{G}-$, lin $\mathrm{A}-$, mefA-, and msrSA-positive strains, respectively (Table S3B). There was no strict correlation between the presence of these genes and MIC values for clindamycin (Table S3A).

\section{Tetracycline Resistance Genes}

Only one strain from Japan (GAI20143) was found to carry tet $\mathrm{X} 1$ (Table 3 ). This strain also harbored tet $\mathrm{Q}$ and
tetX. MIC values of GAI20143 for tetracycline and minocycline were $32 \mu \mathrm{g} / \mathrm{mL}$ and $4 \mu \mathrm{g} / \mathrm{mL}$, respectively. Five other strains (two from Vietnam and three from Japan) harbored either tet $\mathrm{Q}$ or tet $\mathrm{X}$ and showed phenotypic susceptibility to tetracycline (Figure 2A). In contrast, eight strains did not carry any of the tested resistance genes but showed MIC values for tetracycline $>16 \mu \mathrm{g} / \mathrm{mL}$ and for minocycline $>2 \mu \mathrm{g} / \mathrm{mL}$. Interestingly, these strains were isolated from Vietnam (Figure 2A and B).

\section{Fluoroquinolone Resistance Genes}

This study investigated the presence of four genes related to fluoroquinolones resistance in Bacteroides (bexA, qnrA, $q n r B$, qnr $S$ ). The prevalence of those genes is shown in Table 3. The qnrB and qnrS genes were found in Vietnamese strains only. No strain was found to carry more than one of those genes. The distribution of bex $\mathrm{A}$, $q n r \mathrm{~A}, q n r \mathrm{~B}, q n r \mathrm{~S}$ in all strains based on country and levofloxacin and ciprofloxacin MIC values is shown in Figure S1. There was no relationship between those genes and MIC values of both levofloxacin and ciprofloxacin.

\section{Discussion}

Many studies on antibiotic resistance in Bacteroides spp. have been conducted worldwide, but these have mainly focused on clinical isolates (Table S2). B. fragilis, the 


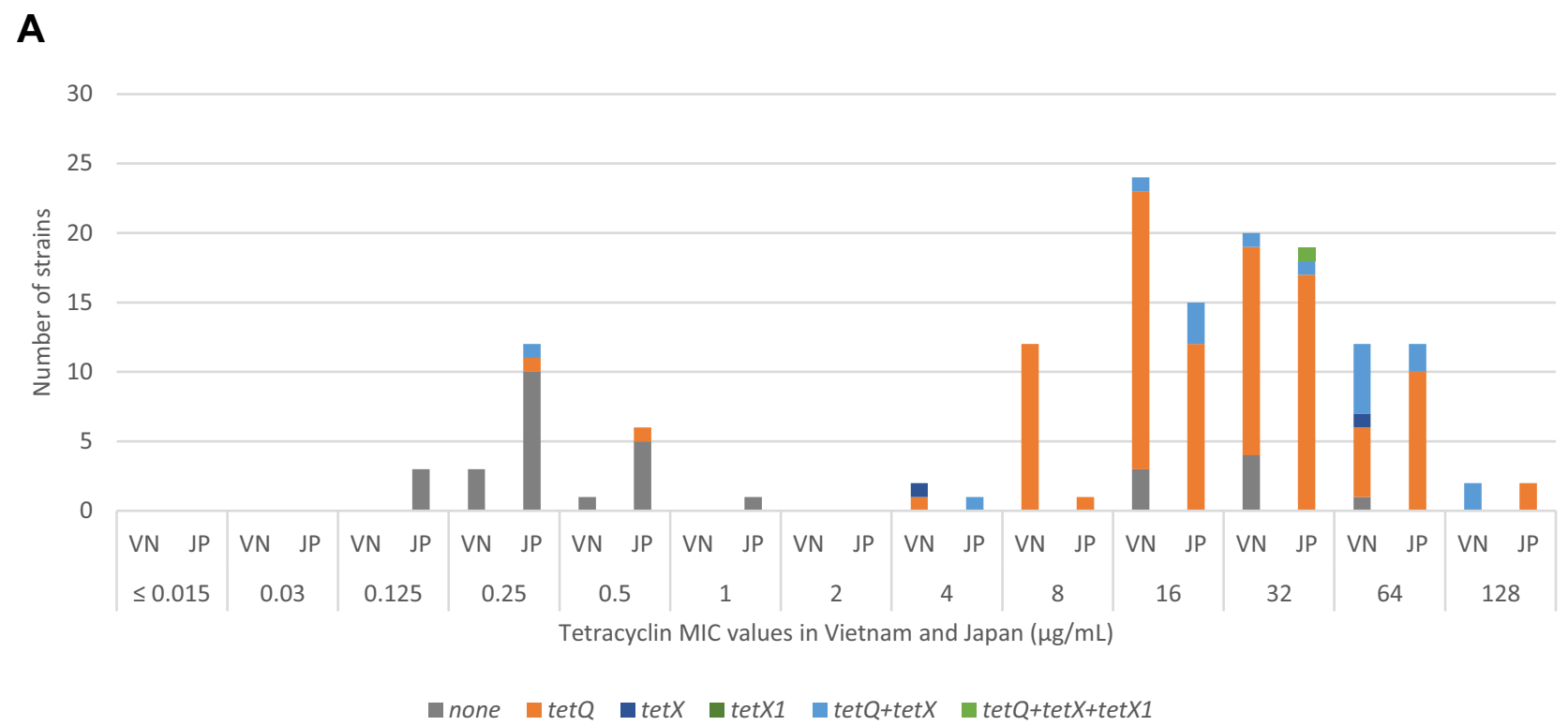

B

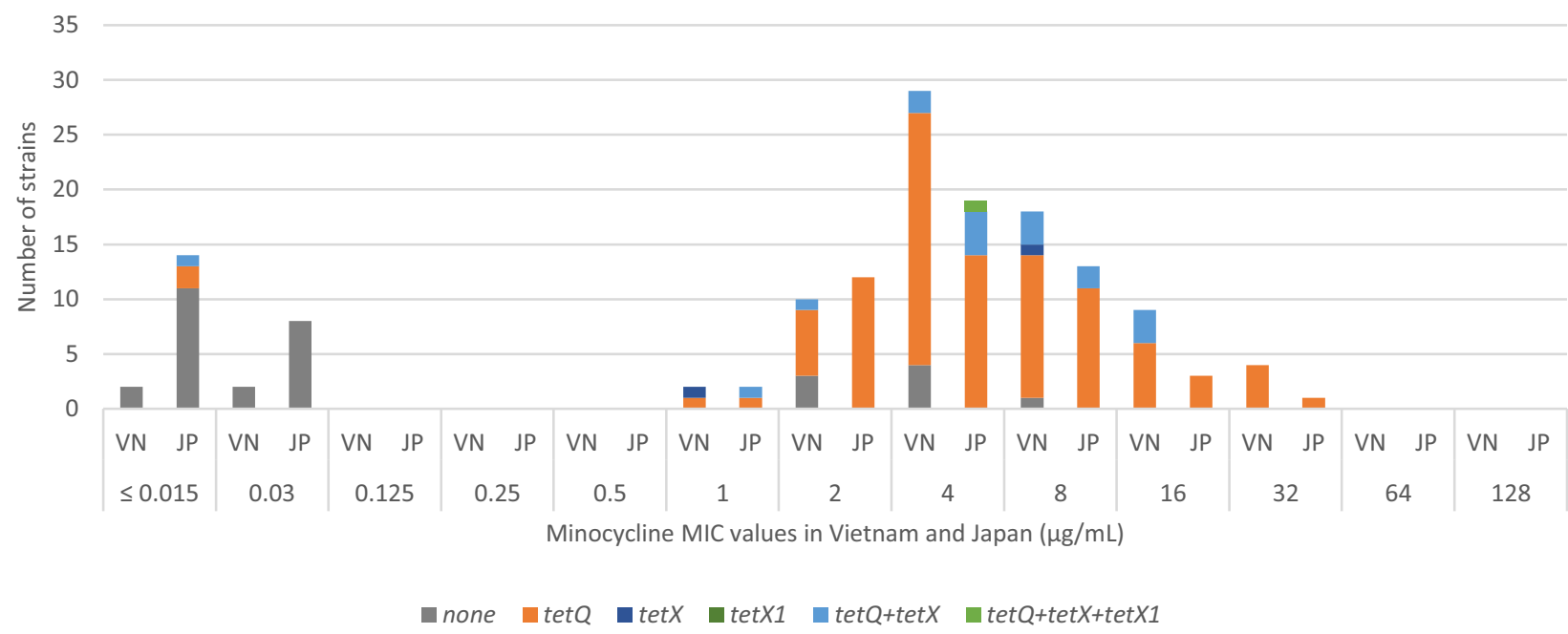

Figure 2 The distribution of resistance genes responsible for tetracycline resistance (tetQ, tetX, tetXI) found in Bacteroides strains in Vietnam and Japan based on MIC values for tetracycline and minocycline. (A) MIC values for tetracycline in Vietnam and Japan $(\mu \mathrm{g} / \mathrm{mL})$. (B) MIC values for minocycline in Vietnam and Japan ( $\mu g / \mathrm{mL})$.

Abbreviations: VN, Vietnam; JP, Japan.

most virulent Bacteroides species, ${ }^{7}$ is the most commonly detected isolate found in clinical samples, but it was not the dominant species identified in the present study. Among studies on fecal samples from healthy individuals, the percentages of $B$. fragilis are inconsistent. Indeed, studies from Brazil, Iran, and Poland reported a relatively high prevalence of $B$. fragilis in healthy individuals compared to our findings (Table $\underline{\mathrm{S} 2}) .{ }^{4,16-18}$ The observed discrepancies might be attributed to different techniques (sampling, culturing technique, number of pick-ups), variations in the recovery rates of bacterial strains, and the biodiversity of human bacterial flora in different people in different countries. In general, B. fragilis is not predominant in BFG. ${ }^{7,19}$ Our study supports this finding.

Thirty-one isolates from Vietnam and 29 from Japan were negative for cepA, $c f x A$, and $c f i$, but all the isolated strains (139) tested positive for production of $\beta$-lactamase. This finding suggests that other types of $\beta$-lactamase production can be found in these isolates. Notably, cepA was reportedly found only in $B$. fragilis as a species-specific $\beta$ lactamase-encoding gene. ${ }^{20-22}$ The cep $A$-positive non- 
fragilis Bacteroides were recently reported in Europe ${ }^{23}$ and in Iran, with a notably higher prevalence. ${ }^{24}$ Our study is the first to document this phenomenon in Vietnam and Japan. In this study, four strains that were highly resistant to cefmetazoles (MIC of $128 \mu \mathrm{g} / \mathrm{mL}$ ) were observed, three of which (two B. thetaiotaomicron from Vietnam and one $B$. fragilis from Japan) harbored neither $c f x \mathrm{~A}$ nor $c f i \mathrm{~A}$. This finding raises concerns regarding cefmetazole resistance in Bacteroides with inconsistent mechanisms, as recently noted in the literature. ${ }^{21,25}$ Of the two non-fragilis Bacteroides ( $P$. distasonis from Vietnam and B. ovatus from Japan) that were moderately resistant to meropenem (MIC values of $16 \mu \mathrm{g} / \mathrm{mL}$ ), $P$. distasonis did not carry c $f(\mathrm{~A}$, but $B$. ovatus did. Notably, six non-fragilis Bacteroides with silent cfiA were found in both Vietnam and Japan. The observation of silent $c f$ A has been previously described. ${ }^{21,23}$ The present study reports two phenomena: cfiA-negative $P$. distasonis resistant to meropenem and occurrence of silent $c f i \mathrm{~A}$ in non-fragilis Bacteroides (Table 3). BFG carbapenem resistance is still rare but has recently been reported worldwide, including Japan. ${ }^{23,26-29}$ The most common mechanism of acquiring resistance to carbapenems in Bacteroides is by producing carbapenemase, a metallo- $\beta$-lactamase enzyme encoded by $c f i$ A. This mechanism mainly contributes to carbapenem resistance in B. fragilis and is quite rare in non-fragilis Bacteroides. Therefore, the wide prevalence of the silent $c f i A$ in non-fragilis Bacteroides, which is more resistant than $B$. fragilis, is a serious concern. The $c f$ A-negative meropenem-resistant $P$. distasonis was resistant to other $\beta$-lactam antibiotics with high MIC values and harbored neither cepA nor $c f x \mathrm{~A}$. We intend to conduct further investigation to better understand these findings.

In this study, metronidazole was highly effective against all isolates, and nim was not found in any of them. Previous studies have shown that the nim gene has not yet circulated among anaerobic bacteria in Japan. ${ }^{20,21}$ In recent reports from India, the nim gene was detected at a notably high prevalence in $\mathrm{BFG}$ isolated from clinical samples. ${ }^{29,30}$ This study is the first surveillance on the presence of nim-carrying anaerobes among isolates from healthy individuals in Vietnam. In Vietnam, metronidazole is widely used to treat anaerobic and parasitic infections; however, metronidazole resistance or circulation of the nim gene has not yet been investigated in Vietnam.

Clindamycin-resistant strains carried $\operatorname{erm} \mathrm{F}, \operatorname{erm} \mathrm{G}, \operatorname{lin} \mathrm{A}$, $m e f \mathrm{~A}$, and $m s r \mathrm{SA}$, but some clindamycin-non-resistant strains also carried these genes. There was no strict correlation between the presence of these genes and MIC values for clindamycin (Table S3A). This observation indicates that the presence of only some of these genes might not be sufficient to lead to phenotypic clindamycin resistance, although their presence in combination might in some cases (Table S3B). Further studies on the expression of these genes will be necessary to define their contribution to antibiotic resistance. Significant differences in the distribution of erm $\mathrm{F}$ and $\operatorname{lin} \mathrm{A}$ between clindamycin-resistant and non-resistant groups were observed in Vietnam but not in Japan (Table S3A). This finding partially supports the hypothesis that $\operatorname{erm} \mathrm{F}$ and $\operatorname{lin} \mathrm{A}$, alone or in combination with other MLS resistance genes, play an important role in the clindamycin-resistance mechanism in $\mathrm{BFG}^{21,23,28,31,32}$ A five-year-analysis from Poland showed a very close correlation between the presence of the ermF gene and MIC values for clindamycin in $\mathrm{BFG} .{ }^{32}$ Considering that erm $\mathrm{F}$ and $\operatorname{lin} \mathrm{A}$ are two of a variety of transmissible elements involved in disseminating antibiotic resistance determinants, this finding addresses a serious concern about the future of clindamycin-based antibiotic therapy, not only for BFG but also other residents of the human flora. ${ }^{6,83}$

Compared to recent studies from European countries on clinical samples, ${ }^{23,34}$ our results, in Vietnam and Japan separately, showed a considerably higher presence of resistance genes $(e r m \mathrm{~F}, e r m \mathrm{G}$, $\operatorname{lin} \mathrm{A}, m e f \mathrm{~A}$, and $m s r \mathrm{SA})$ in both clindamycin-resistant and non-resistant groups (Table S3). Our findings were in agreement with a report from Poland in $2019^{4}$ that BFG bacteria isolated from healthy people carry high frequencies of genes encoding resistance to MLS antibiotics. Although our study detected the five most common MLS resistance genes, many clindamycinresistant strains with higher MIC values did not carry any of those genes, indicating the presence of unknown resistance mechanisms (Table S3B).

Only one strain, B. thetaiotaomicron, carried a set of three genes, erm G-mefA-msrSA. This combination was previously reported in a conjugative transposon, CTnGERM1. ${ }^{35}$ Our isolate was found to be highly resistant to clindamycin, clarithromycin, and erythromycin. Recent findings from European countries showed a notable prevalence of the erm $\mathrm{G}-m e f \mathrm{~A}-m s r \mathrm{SA}$ combination. $^{23}$ However, despite a higher prevalence of resistance genes in the present study, the ermG-mefA-msrSA combination was not common.

Susceptibility tests were performed for all strains with both clarithromycin and erythromycin. The MIC values of clarithromycin and erythromycin showed similarities, but with some exceptions, as listed in Table S4. These strains were found in both the countries. There were no notable 
differences in the resistance genes among the strains. Therefore, using MIC values for erythromycin to interpret clarithromycin susceptibility status may cause some misleading results. With such diversity in susceptibility distribution, a holistic approach is needed in the future to test the susceptibility of BFG to macrolides.

Five tetracycline-susceptible strains harbored either tet $\mathrm{Q}$ or tet $\mathrm{X}$. In contrast, eight tetracycline-resistant strains from Vietnam did not carry any of the tested resistance genes (Figure 2A). This raises a question about the possible existence of silent tet $\mathrm{Q}$ and tetX. Another issue that can be considered is that Vietnam, with its over-the-counter antibiotic use, might be nurturing a variety of mechanisms for tetracyclineresistance among $\mathrm{BFG}$, such as drug efflux (tetA-E, tet $\mathrm{K}-\mathrm{L})$ or ribosomal protection $(\operatorname{tet} \mathrm{M}, \operatorname{tet} \mathrm{W})$, such as those that have been found in recent publications. ${ }^{4,36}$ Even though tetracycline is not advised to treat BFG infections, the mechanisms by which a strain can become resistant pose a serious concern in anaerobic research because of the potential for transfer to other human flora via plasmids and transposons, especially under conditions in which there is exposure to antibiotics. ${ }^{8,33,36,37}$

The use of quinolones as monotherapy for mixed infections has been limited by their lack of activity against anaerobic pathogens, especially in the case of ciprofloxacin. ${ }^{33,38}$ However, newly developed fluoroquinolones, such as the fourth- and fifth-generation fluoroquinolones, showed promising effects in treating BFG infection. ${ }^{38-40}$ Moreover, recent studies of fluoroquinolone-resistance in which qnrA, $q n r \mathrm{~B}, q n r \mathrm{~S}$, and bex $\mathrm{A}$ were commonly detected also concluded that an effective method for determining fluoroquinolone-resistant mechanisms remains to be developed. ${ }^{4,23}$ Further analysis of mutations in gyrA needs to be carried out on these strains to better understand this issue.

Differences in antibiotic-resistant patterns and prevalence of resistance genes between countries might be caused by differences in how antibiotics are used in those countries. Some studies on aerobic bacteria in healthy people in Vietnam and Japan also showed a high prevalence of resistance genes. ${ }^{13,14,41}$ Further investigations on the relationship between antibiotic usage and the prevalence of resistance genes in the human flora, both aerobic and anaerobic, will need to be carried out.

\section{Conclusion}

This study is the first to genetically and phenotypically characterize antimicrobial resistance of Bacteroides isolated from healthy individuals in Vietnam and Japan. Data collected revealed the need to broaden the focus of BFG research to include not only the most virulent member B. fragilis, but also other members owing to their overwhelming numbers and their role as a source of diverse antibiotic-resistant determinants.

Isolates from Vietnam and Japan showed similarities in antimicrobial-resistant BFG. However, isolates from Vietnam showed significantly higher resistance to piperacillin, cefmetazole, clindamycin, tetracycline, and minocycline.

A comparison of the predominant species in the healthy population of these two Asian countries showed several differences but further research will be required to investigate and extend the suggestive findings of this work.

\section{Abbreviations}

BFG, Bacteroides fragilis group; MALDI-TOF MS, matrix-assisted laser desorption ionization - time of flight mass spectrometry; MIC, minimum inhibitory concentration; MLS, macrolide-lincomycin-streptogramin; PCR, polymerase chain reaction.

\section{Ethics Approval and Informed Consent}

The study was approved by the Ethics Committees of Gifu University (Gifu, Japan; Approval Number 2019-164) and Thai Binh University of Medicine and Pharmacy (Thai Binh, Vietnam; Approval Number 773.1/HĐÐÐ). All study participants provided informed written consent prior to study enrollment. This study was conducted in accordance with the Declaration of Helsinki.

\section{Acknowledgments}

We thank Ms. Akiko Nakagawa for her technical support in the susceptibility testing. We also thank Professor Yoshinori Muto for his advice on our manuscript preparation.

\section{Author Contributions}

All authors made a significant contribution to the work reported, whether that is in the conception, study design, execution, acquisition of data, analysis and interpretation, or in all these areas; took part in drafting, revising or critically reviewing the article; gave final approval of the version to be published; have agreed on the journal to which the article has been submitted; and agree to be accountable for all aspects of the work. 


\section{Funding}

This work was supported by the Japan Society for the Promotion of Science KAKENHI (grant number 20H00561).

\section{Disclosure}

The authors report no conflicts of interest.

\section{References}

1. Nagy E, Urban E, Nord CE; ESCMID Study Group on Antimicrobial Resistance in Anaerobic Bacteria. Antimicrobial susceptibility of Bacteroides fragilis group isolates in Europe: 20 years of experience. Clin Microbiol Infect. 2011;17(3):371-379. doi:10.1111/ j.1469-0691.2010.03256.x

2. Hecht DW. Prevalence of antibiotic resistance in anaerobic bacteria: worrisome developments. Clin Infect Dis. 2004;39(1):92-97. doi: $10.1086 / 421558$

3. Galvao BP, Meggersee RL, Abratt VR. Antibiotic resistance and adhesion potential of Bacteroides fragilis clinical isolates from Cape Town, South Africa. Anaerobe. 2011;17(4):142-146. doi:10.1016/j.anaerobe.2011.02.009

4. Niestepski S, Harnisz M, Korzeniewska E, et al. The emergence of antimicrobial resistance in environmental strains of the Bacteroides fragilis group. Environ Int. 2019;124:408-419. doi:10.1016/j. envint.2018.12.056

5. Kierzkowska M, Majewska A, Mlynarczyk G. Trends and impact in antimicrobial resistance among bacteroides and parabacteroides species in 2007-2012 compared to 2013-2017. Microb Drug Resist. 2020;26(12):1452-1457. doi:10.1089/mdr.2019.0462

6. Kierzkowska M, Majewska A, Szymanek-Majchrzak K, SawickaGrzelak A, Mlynarczyk A, Mlynarczyk G. In vitro effect of clindamycin against Bacteroides and Parabacteroides isolates in Poland. $J$ Glob Antimicrob Resist. 2018;13:49-52. doi:10.1016/j.jgar.2017.11.001

7. Wexler HM. Bacteroides: the good, the bad, and the nitty-gritty. Clin Microbiol Rev. 2007;20(4):593-621. doi:10.1128/CMR.00008-07

8. Whittle G, Shoemaker NB, Salyers AA. The role of Bacteroides conjugative transposons in the dissemination of antibiotic resistance genes. Cell Mol Life Sci. 2002;59(12):2044-2054. doi:10.1007/ s000180200004

9. Shoemaker NB, Vlamakis H, Hayes K, Salyers AA. Evidence for extensive resistance gene transfer among Bacteroides spp. and among bacteroides and other genera in the human colon. Appl Environ Microbiol. 2001;67(2):561-568. doi:10.1128/AEM.67.2.561-568.2001

10. Stentz R, Horn N, Cross K, et al. Cephalosporinases associated with outer membrane vesicles released by Bacteroides spp. protect gut pathogens and commensals against beta-lactam antibiotics. $J$ Antimicrob Chemother. 2015;70(3):701-709. doi:10.1093/jac/ dku466

11. Nahui Palomino RA, Vanpouille C, Costantini PE, Margolis L. Microbiota-host communications: bacterial extracellular vesicles as a common language. PLoS Pathog. 2021;17(5):e1009508. doi:10.1371/journal.ppat.1009508

12. Bui TM, Hirai I, Ueda S, et al. Carriage of Escherichia coli producing CTX-M-type extended-spectrum beta-lactamase in healthy vietnamese individuals. Antimicrob Agents Chemother. 2015;59 (10):6611-6614. doi:10.1128/AAC.00776-15

13. Yamamoto Y, Kawahara R, Fujiya Y, et al. Wide dissemination of colistin-resistant Escherichia coli with the mobile resistance gene mor in healthy residents in Vietnam. J Antimicrob Chemother. 2019;74 (2):523-524. doi:10.1093/jac/dky435

14. Kawahara R, Fujiya Y, Yamaguchi T, et al. Most domestic livestock possess colistin-resistant commensal Escherichia coli harboring mcr in a rural community in Vietnam. Antimicrob Agents Chemother. 2019;63(6). doi:10.1128/AAC.00594-19
15. Clinical and Laboratory Standards Institute. Methods for Antimicrobial Susceptibility Testing for Anaerobic Bacteria, CLSI Standard M11 (Ninth Ed.). Clinical and Laboratory Standards Institute; 2018.

16. Nakano V, Avila-Campos MJ. Survey of antimicrobial susceptibility patterns of the bacteria of the Bacteroides fragilis group isolated from the intestinal tract of children. Mem Inst Oswaldo Cruz. 2004;99 (3):319-324. doi:10.1590/S0074-02762004000300014

17. Boente RF, Ferreira LQ, Falcao LS, et al. Detection of resistance genes and susceptibility patterns in Bacteroides and Parabacteroides strains. Anaerobe. 2010;16(3):190-194. doi:10.1016/j.anaerobe.2010.02.003

18. Narimani T, Douraghi M, Owlia P, et al. Heterogeneity in resistant fecal Bacteroides fragilis group collected from healthy people. Microb Pathog. 2016;95:1-6. doi:10.1016/j.micpath.2016.02.017

19. Kulagina EV, Efimov BA, Maximov PY, Kafarskaia LI, Chaplin AV, Shkoporov AN. Species composition of Bacteroidales order bacteria in the feces of healthy people of various ages. Biosci Biotechnol Biochem. 2012;76(1):169-171. doi:10.1271/bbb.110434

20. Ogane K, Tarumoto N, Kodana M, et al. Antimicrobial susceptibility and prevalence of resistance genes in Bacteroides fragilis isolated from blood culture bottles in two tertiary care hospitals in Japan. Anaerobe. 2020;64:102215. doi:10.1016/j.anaerobe.2020.102215

21. Tran CM, Tanaka K, Watanabe K, Tanaka K, Watanabe K. PCRbased detection of resistance genes in anaerobic bacteria isolated from intra-abdominal infections. $J$ Infect Chemother. 2013;19 (2):279-290. doi:10.1007/s10156-012-0532-2

22. Parker AC, Smith CJ. Genetic and biochemical analysis of a novel Ambler class A beta-lactamase responsible for cefoxitin resistance in Bacteroides species. Antimicrob Agents Chemother. 1993;37 (5):1028-1036. doi:10.1128/AAC.37.5.1028

23. Eitel Z, Sóki J, Urbán E, Nagy E. The prevalence of antibiotic resistance genes in Bacteroides fragilis group strains isolated in different European countries. Anaerobe. 2013;21:43-49. doi:10.1016/j.anaerobe.2013.03.001

24. Kouhsari E, Mohammadzadeh N, Kashanizadeh MG, et al. Antimicrobial resistance, prevalence of resistance genes, and molecular characterization in intestinal Bacteroides fragilis group isolates. APMIS. 2019;127(6):454-461. doi:10.1111/apm.12943

25. Liu CY, Huang YT, Liao CH, Yen LC, Lin HY, Hsueh PR. Increasing trends in antimicrobial resistance among clinically important anaerobes and Bacteroides fragilis isolates causing nosocomial infections: emerging resistance to carbapenems. Antimicrob Agents Chemother. 2008;52(9):3161-3168. doi:10.1128/AAC.00355-08

26. Ulger Toprak N, Akgul O, Bilgin H, et al. Frequency and associated factors for carbapenem-non-susceptible Bacteroides fragilis group bacteria colonization in hospitalized patients: case control study in a university hospital in Turkey. Indian $J$ Med Microbiol. 2021;39:518-522. doi:10.1016/j.ijmmb.2021.03.018

27. Takesue Y, Kusachi S, Mikamo H, et al. Antimicrobial susceptibility of common pathogens isolated from postoperative intra-abdominal infections in Japan. $J$ Infect Chemother. 2018;24(5):330-340. doi:10.1016/j.jiac.2018.02.011

28. Jasemi S, Emaneini M, Ahmadinejad Z, et al. Antibiotic resistance pattern of Bacteroides fragilis isolated from clinical and colorectal specimens. Ann Clin Microbiol Antimicrob. 2021;20(1):27. doi:10.1186/s12941-021-00435-w

29. Sood A, Ray P, Angrup A. Phenotypic and genotypic antimicrobial resistance in clinical anaerobic isolates from India. JAC Antimicrob Resist. 2021;3(2):dlab044. doi:10.1093/jacamr/dlab044

30. Sethi S, Shukla R, Bala K, Gautam V, Angrup A, Ray P. Emerging metronidazole resistance in Bacteroides spp. and its association with the nim gene: a study from North India. J Glob Antimicrob Resist. 2019;16:210-214. doi:10.1016/j.jgar.2018.10.015

31. Veloo ACM, Baas WH, Haan FJ, Coco J, Rossen JW. Prevalence of antimicrobial resistance genes in Bacteroides spp. and Prevotella spp. Dutch clinical isolates. Clin Microbiol Infect. 2019;25(9):1156 e1159-1156 e1113. doi:10.1016/j.cmi.2019.02.017 
32. Kierzkowska M, Majewska A, Szymanek-Majchrzak K, SawickaGrzelak A, Mlynarczyk A, Mlynarczyk G. The presence of antibiotic resistance genes and bft genes as well as antibiotic susceptibility testing of Bacteroides fragilis strains isolated from inpatients of the Infant Jesus Teaching Hospital, Warsaw during 2007-2012. Anaerobe. 2019;56:109-115. doi:10.1016/j.anaerobe.2019.03.003

33. Gajdacs M, Spengler G, Urban E. Identification and antimicrobial susceptibility testing of anaerobic bacteria: rubik's cube of clinical microbiology? Antibiotics (Basel). 2017;6(4). doi:10.3390/ antibiotics 6040025

34. Rong SMM, Rodloff AC, Stingu CS. Diversity of antimicrobial resistance genes in Bacteroides and Parabacteroides strains isolated in Germany. $J$ Glob Antimicrob Resist. 2021;24:328-334. doi:10.1016/j.jgar.2021.01.007

35. Wang Y, Wang GR, Shelby A, Shoemaker NB, Salyers AA. A newly discovered Bacteroides conjugative transposon, CTnGERM1, contains genes also found in gram-positive bacteria. Appl Environ Microbiol. 2003;69(8):4595-4603. doi:10.1128/AEM.69.8.4595-4603.2003

36. Jung JY, Ahn Y, Khare S, Gokulan K, Pineiro SA, Cerniglia CE. An in vitro study to assess the impact of tetracycline on the human intestinal microbiome. Anaerobe. 2018;49:85-94. doi:10.1016/j. anaerobe.2017.12.011
37. Wu L, Xie X, Li Y, et al. Metagenomics-based analysis of the age-related cumulative effect of antibiotic resistance genes in gut microbiota. Antibiotics (Basel). 2021;10(8):1006.

38. Snydman DR, Jacobus NV, McDermott LA, et al. In vitro activities of newer quinolones against bacteroides group organisms. Antimicrob Agents Chemother. 2002;46(10):3276-3279. doi:10.1128/AAC.46.1 0.3276-3279.2002

39. Tanaka K, Vu H, Hayashi M. In vitro activities and spectrum of lascufloxacinKRP-AM1977against anaerobes. J Infect Chemother. 2021;27(8):1265-1269. doi:10.1016/j.jiac.2021.03.026

40. Rusu A, Lungu IA, Moldovan OL, Tanase C, Hancu G. Structural characterization of the millennial antibacterial (fluoro)quinolones-shaping the fifth generation. Pharmaceutics. 2021;13(8):1289. doi:10.3390/pharmaceutics 13081289

41. Luvsansharav UO, Hirai I, Niki M, et al. Prevalence of fecal carriage of extended-spectrum beta-lactamase-producing Enterobacteriaceae among healthy adult people in Japan. J Infect Chemother. 2011;17 (5):722-725. doi:10.1007/s10156-011-0225-2
Infection and Drug Resistance

\section{Publish your work in this journal}

Infection and Drug Resistance is an international, peer-reviewed openaccess journal that focuses on the optimal treatment of infection (bacterial, fungal and viral) and the development and institution of preventive strategies to minimize the development and spread of resistance. The journal is specifically concerned with the epidemiology of
Dovepress

antibiotic resistance and the mechanisms of resistance development and diffusion in both hospitals and the community. The manuscript management system is completely online and includes a very quick and fair peerreview system, which is all easy to use. Visit http://www.dovepress.com/ testimonials.php to read real quotes from published authors. 